\title{
Four Narcolepsy Cases after H1N1 Infection or Vaccination
}

\author{
Tae Won Kim, MD, Hye Jin Kim, MD, Dong Woo Kang, MD, Hyun Jung Kwak, MD, Seung Chul Hong, MD, PhD \\ Department of Psychiatry, St. Vincent's Hospital, College of Medicine, The Catholic University of Korea, Suwon, Korea
}

\begin{abstract}
This report describes 4 narcolepsy cases that were developed after Influenza A/H1N1 infection or vaccination. The durations from Influenza A/H1N1 infection or vaccination to the symptom onset were 10 days, 2 weeks, 3 months, 6 months respectively. Among the four, two were teenagers or younger. One of them developed symptoms after Influenza A/H1N1 infection while three of them developed after vaccination. All went through polysomnography followed by multiple sleep latency tests for accurate diagnosis. All suffered from cataplexy. However, one of them presented with negative results in the human leukocyte antigen DQB1*0602 study. These cases suggest a possibility an association between H1N1/Influenza A virus and narcolepsy development via immune mechanism.
\end{abstract}

Sleep Med Res 2015;6(1):38-41

Key Words Narcolepsy, H1N1 influenza, Vaccination, Human leukocyte antigen-DQB1*0602, Cataplexy.

Received: December 1, 2014

Revised: January 15, 2015

Accepted: April 8, 2015

Correspondence

Seung Chul Hong, MD, PhD

Department of Psychiatry,

St. Vincent's Hospital, College of Medicine,

The Catholic University of Korea,

93 Jungbu-daero, Paldal-gu, Suwon

442-723, Korea

Tel +82-31-249-7114

Fax +82-31-248-6758

E-mail hscjohn@hotmail.com

\section{INTRODUCTION}

Narcolepsy was known as a disease caused by loss of hypocretin cells in the hypothalamus, might be an autoimmune disorder or involve an infectious agent with participation of immune system. ${ }^{1}$ The diagnostic criteria of narcolepsy according to the third edition of International Classification of Sleep Disorders-3 is: 1) The patient has daily periods of irrepressible need to sleep or daytime lapses into sleep occurring for at least three months. 2) The presence of one or both of the following: (1) Cataplexy (as defined under Essential Features) and a mean sleep latency of $\leq 8$ minutes and two or more sleep onset rapid eye movement (REM) periods (SOREMPs) on an multiple sleep latency tests (MSLT) performed according to standard techniques. A SOREMP (within 15 minutes of sleep onset) on the preceding nocturnal polysomnogram may replace one of the SOREMPs on the MSLT. (2) Cerebrospinal fluid (CSF) hypocretin- 1 concentration, measured by immunoreactivity, is either $\leq 110 \mathrm{pg} / \mathrm{mL}$ or $<1 / 3$ of mean values obtained in normal subjects with the same standardized assay. The mechanism seems to be associated with human leukocyte antigen (HLA), T-cell receptor polymorphisms and recently reported Tribbles 2 autoantibodies. ${ }^{2}$ However, only 1-2\% of first degree relatives of narcoleptics develop the disease and 70-75\% of monozygotic twins are discordant for the disorder, suggesting strong environmental influence. ${ }^{3,4}$ Previous studies proposed Streptococcal infections as probable environmental trigger for narcolepsy. ${ }^{5}$ We report 4 cases of narcolepsy that were developed after Influenza A/H1N1 infection or vaccination who had neither previous medicosurgical nor neuropsychiatric history.

\section{CASE REPORT}

\section{Case 1: A 42-Year-Old Female Patient}

The patient is a homemaker. Her daughter is a 16-year-old student who has been suffering from excessive daytime sleepiness since she was 9 years old. In late November of 2009, she 
was diagnosed with Influenza A/H1N1 and prescribed Tamiflu. After 10 days, symptoms of excessive daytime sleepiness appeared. Starting early 2010, she presented with cataplexy that lasted from 2 to 10 minutes, predominantly precipitated by anger. These symptoms usually resolved after a nap. In February 2011, she visited the sleep center at St. Vincent's Hospital. The immunological evaluation (Helicobacter Pylori Ab, IgG) was normal and HLA typing (HLA-DQB1*0602) was negative. Epworth Sleepiness Scale (ESS) score was 18 ( $\geq 16$, severe daytime sleepiness).

A polysomnography (PSG) revealed a total sleep time of 482.9 minutes, normal nocturnal breathing, and 37 periodic limb movements. A MSLT followed, which showed a mean sleep latency of 2.1 minutes and 2 SOREMPs during the 5 naps. We diagnosed her as narcolepsy with cataplexy. $100 \mathrm{mg}$ of modafinil per day was prescribed. Her sleepiness and domestic performance improved dramatically. After a month, her ESS was decreased to 4 .

\section{Case 2: A 14-Year-Old Male Patient}

The patient's father has suffered from excessive daytime sleepiness since he was 15 years old. In early December 2009, the patient was vaccinated against the 2009 pandemic Influenza A (H1N1) virus (A/California/7/2009). After 6 months, he started to suffer from abrupt onset of excessive daytime sleepiness, nightmares, sleep paralysis, hypnagogic hallucination and poor quality of sleep. He also presented with cataplexy that lasted from 2 to 10 minutes, predominantly precipitated by exercising and laughing. In early in August of 2010 he visited the sleep center at St. Vincent's Hospital. The immunological evaluation (Helicobacter Pylori Ab, IgG and anti-Streptococcal antibodies) was normal but HLA typing (HLA-DQB1*0602) was positive. ESS was 18.

A PSG revealed a total sleep time of 341.5 minutes, normal nocturnal breathing, and 91 periodic limb movements. A MSLT followed, which showed a mean sleep latency of 0.3 minutes and 3 SOREMPs during the 5 naps. We diagnosed him as narcolepsy with cataplexy. $10 \mathrm{mg}$ of methylphenidate and 75 mg venlafaxine per day were prescribed. His periodic sleepiness, cataplexy and poor school performance persisted. We increased dose of methylphenidate to $15 \mathrm{mg}$ /day and venlafaxine to $150 \mathrm{mg}$ /day. However his symptoms had not improved.

\section{Case 3: A 29-Year-Old Male Patient}

The patient was in an unemployed status. His other family members had no problem related to sleep. In mid-November 2010, He was vaccinated against the pandemic Influenza A (H1N1) virus with FluMist (Influenza A H1N1/California/2009), an intranasal spray, in Korea. After 2 weeks from the vaccination, he presented with an abrupt onset of excessive daytime sleepiness, nightmares, sleep paralysis, hypnagogic hallucination and a poor quality of sleep. He also presented with cataplexy, predominantly precipitated by exercise and laughing. In July 2011, he visited the sleep center at St. Vincent's Hospital. The immunological evaluation (Helicobacter Pylori Ab, IgG and anti-Streptococcal antibodies) was normal but HLA typing (HLA-DQB1*0602) was positive. ESS was 21.

A PSG showed a total sleep time of 438.5 minutes, normal nocturnal breathing, and 76 periodic limb movements. A MSLT showed a mean sleep latency of 0.1 minutes and 4 SOREMPs during the 4 naps. We diagnosed him as narcolepsy with cataplexy. $200 \mathrm{mg}$ /day of modafinil and $150 \mathrm{mg} /$ day of venlafaxine were prescribed. However, daytime sleepiness, cataplexy and sleep paralysis exacerbated after he was vaccinated again 3-4 months later with Green-Cross Flu. The dosage of modafinil was increased to $400 \mathrm{mg} /$ day, venlafaxine to $225 \mathrm{mg} /$ day and $10 \mathrm{mg} /$ day of methylphenidate, $25 \mathrm{mg} /$ day of trazodone, $0.5 \mathrm{mg} /$ day of clonazepam were added. Even after increasing the dosage, not only his symptoms persisted, but also headache, derealization, and cognitive impairment accompanied. Eventually in July 2012, he had to quit the job due to severe cataplexy.

\section{Case 4: A 9-Year-Old Female Patient}

The patient received vaccination (Green-Cross flu; Green Cross Corp., Yongin, Korea) against H1N1 influenza virus on November 26th, 2009. There was no side effect observed such as fever or seizure except for mild pain at the injection site. However, after 3 months from the vaccination, she started to fall asleep during classes, which she never had experienced before. Teachers at school could notice that the patient was suffering from excessive daytime sleepiness. When riding on public transportation, the patient fell asleep as soon as she got seated (it only took a few minutes) and was not able to wake up at the right station she had to get off even when her friends tried to wake her up by shaking her. After 5 months from vaccination, she experienced drooping eyelids and sudden loss of muscle tension on both lower extremities. The patient suddenly plunked down and could not move for about 30 seconds soon after she started laughing out loud while watching a comedy on television. In addition, she experienced transient muscle weakness in a bad mood such as during an argument with her sister. Excessive daytime sleepiness became severe and sudden loss of muscle tone appeared more frequently in progression. She visited the Department of Pediatrics for evaluation suspicious of epilepsy and myasthenia gravis. She went through 24-hour electroencephalography (EEG) and jolly test, however, the studies showed a negative result. During the 24-hour EEG monitoring sudden neck drop and limping movement of both extremities were recorded on video for several times. However, they happened in the absence of changes in the EEG waves. In speculation of this movement as cataplexy, the patient was referred to the department of psychiatry for diagnosis of narcolepsy. She did not have any medicosurgical history or any other neuropsychiatric disease. The immunological evaluation (Heli- 
cobacter Pylori Ab, IgG and anti-Streptococcal antibodies) was normal but HLA typing (HLA-DQB1*0602) was positive.

A MSLT showed a mean sleep latency of 1.8 minutes and 4 SOREMPs during the 4 naps. We diagnosed her as narcolepsy with cataplexy. $200 \mathrm{mg} /$ day of modafinil and $10 \mathrm{mg} /$ day of clomipramine were prescribed. During treatment, her cataplexy improved in terms of frequency, however, the patient continued to suffer from excessive daytime sleepiness showing little improvement.

\section{DISCUSSION}

In three major centers of reference for narcolepsy-Montpellier, France; Montreal, Canada; and Stanford, United States, 14 post-vaccination cases were identified and 2 cases following H1N1 infection were also reported. Of the 14 post-vaccination cases, only one case reported to have been vaccinated with an intranasal spray, with no adjuvant, just like our third case of 29 -year old male patient. ${ }^{6}$ In Finland, there was a 17 -fold increase in the annual incidence of narcolepsy in 2010 in children aged under 17, following the $2009 \mathrm{H} 1 \mathrm{~N} 1$ pandemic vaccination campaign. It was speculated that Pandemrix vaccination contributed to the increased incidence of narcolepsy in HLA-DQB1*0602 positive children. ${ }^{7}$ The Swedish Medical Product agency in 2011 reported that overall the incidence rate in those vaccinated with Pandemrix was 4.2 versus 0.64 in unvaccinated per 100000 person-years, respectively suggesting a relative risk of 6.6 (95\% confidence interval 3.1-14.5) and an absolute risk of 3.6 (95\% confidence interval $2.5-4.7){ }^{8}$

Then how can one explain the mechanism that $\mathrm{H} 1 \mathrm{~N} 1$ vaccination or infection triggers narcolepsy? Possible hypotheses include a specific immune-mimicry component, mediated through the presentation by DQB1*0602 of a particular autoantigen to a specific $\mathrm{T}$ cell receptor idiotype or generalized stimulation of the immune system by nonspecific factors, such as adjuvants, influenza or Streptococcal infections and other factors. ${ }^{9}$ Infection could act as a nonspecific immune trigger, for example via superantigen stimulation of dormant autoreactive T-cell clones. It is possible that these factors increase brain inflammation or blood brain penetration nonspecifically, allowing the autoimmune process to reach hypocretin cells. Fever and the administration of various adjuvants have been reported to increase blood brain penetration and activate brain microglia in animal models. ${ }^{10}$

Pandemrix is a split-virion inactivated vaccine containing A/ California/7/2009 derived H1, N1 and Polymerase basic 1 antigens re-assorted into a A Puerto Rico/8/34 backbone strain and adjuvanted with a proprietary adjuvant, Adjuvant System 03. ${ }^{9} \mathrm{~A}$ recent review of Scandinavian pharmacovigilance data following pandemic vaccination with other vaccines either unadjuvanted or containing another squalene-based adjuvant (MF59) did not reveal increased narcolepsy. ${ }^{11}$ Additionally, Pandemrix has not been used in Korea. Instead, a different type of non-adjuvanted vaccine was injected and there was no increase in narcolepsy cases during 2009-2010 in Korea.

The fact that data are less clear with vaccines other than Pandemrix suggests that the combination of the nonspecific immune-stimulating effects of adjuvant and of the H1N1 antigen may be important. The presented four cases occurred and exacerbated after Influenza A/H1N1 vaccinations or infection. The clinical and neurophysiologic features, plus the HLA genotype, even without cerebrospinal fluid hypocretin data, fulfill the diagnosis of narcolepsy. Considering the temporal association between the vaccination or infection and the emergence of the clinical symptoms, there may be a link with the vaccine or infection. However, in Chinese data, narcolepsy onset is highly correlated with seasonal and annual patterns of upper airway infections, including H1N1 influenza but not H1N1 vaccination itself. ${ }^{9}$ Therefore, it seems hazardous to withhold vaccination just because of a possible link that might exist or not. And future studies are warranted to understand how a vaccination/adjuvant and other triggers can cause narcolepsy.

There were two case, Case 3 and 4, which were purely absent of family history of hypersomnia. Genetic factor is one of the risk factors of narcolepsy. Therefore, in Case 1 (daughter positive of excessive daytime sleepiness) and in Case 2 (history of excessive daytime sleepiness in patient's father), it could be difficult to explain the pathogenesis is only due H1N1 infection or vaccination. The usual age at onset of narcolepsy is in the second or third decade. Narcolepsy symptoms are often reported to start in childhood or adolescence. Case 3 and 4 had occurred in age 14, 29 which overlaps with the typical age of onset. Therefore, it is difficult to rule out the possibility of incidental occurrence of narcolepsy being interlinked with the time of vaccination. It is interesting that patients of case 1 was negative of HLA typing (HLA-DQB1*0602) when most of the cases reported until now show positive results. There are nine narcolepsy cases reported that was DQB1*0602 negative with low CSF hypocretin. ${ }^{12}$ This suggest the existence of other environmental factors in developing narcolepsy. The rare subtype DPB1*0901, and homologous DPB1*1001 subtype, were present in 5 subjects, suggesting a secondary association with HLADP. ${ }^{12}$ Although we did not have a chance for further work-up to investigate the association, one HLA-negative cases of our report might also be related to the presence of rare subtypes.

\section{Conflicts of Interest}

The authors have no financial conflicts of interest.

\section{REFERENCES}

1. Longstreth WT Jr, Koepsell TD, Ton TG, Hendrickson AF, van Belle G. The epidemiology of narcolepsy. Sleep 2007;30:13-26.

2. Cvetkovic-Lopes V, Bayer L, Dorsaz S, Maret S, Pradervand S, Dauvilliers Y, et al. Elevated Tribbles homolog 2-specific antibody levels in 
narcolepsy patients. J Clin Invest 2010;120:713-9.

3. Guilleminault C, Mignot E, Grumet FC. Familial patterns of narcolepsy. Lancet 1989;2:1376-9.

4. Mignot E. Genetic and familial aspects of narcolepsy. Neurology 1998; 50(2 Suppl 1):S16-22.

5. Aran A, Lin L, Nevsimalova S, Plazzi G, Hong SC, Weiner K, et al. Elevated anti-streptococcal antibodies in patients with recent narcolepsy onset. Sleep 2009;32:979-83.

6. Dauvilliers Y, Montplaisir J, Cochen V, Desautels A, Einen M, Lin L, et al. Post-H1N1 narcolepsy-cataplexy. Sleep 2010;33:1428-30.

7. Partinen M, Saarenpää-Heikkilä O, Ilveskoski I, Hublin C, Linna M, Olsén $\mathrm{P}$, et al. Increased incidence and clinical picture of childhood narcolepsy following the $2009 \mathrm{H} 1 \mathrm{N1}$ pandemic vaccination campaign in Finland. PLoS One 2012;7:e33723.

8. Eurosurveillance editorial team. Swedish Medical Products Agency publishes report from a case inventory study on Pandemrix vaccination and development of narcolepsy with cataplexy. Euro Surveill 2011;16. pii: 19904.

9. Han F, Lin L, Warby SC, Faraco J, Li J, Dong SX, et al. Narcolepsy onset is seasonal and increased following the $2009 \mathrm{H} 1 \mathrm{~N} 1$ pandemic in China. Ann Neurol 2011;70:410-7.

10. Banks WA, Erickson MA. The blood-brain barrier and immune function and dysfunction. Neurobiol Dis 2010;37:26-32.

11. Tsai TF, Crucitti A, Nacci P, Nicolay U, Della Cioppa G, Ferguson J, et al. Explorations of clinical trials and pharmacovigilance databases of MF59 ${ }^{\circledR}$-adjuvanted influenza vaccines for associated cases of narcolepsy. Scand J Infect Dis 2011;43:702-6.

12. Han F, Lin L, Schormair B, Pizza F, Plazzi G, Ollila HM, et al. HLA DQB1*06:02 negative narcolepsy with hypocretin/orexin deficiency. Sleep 2014;37:1601-8. 